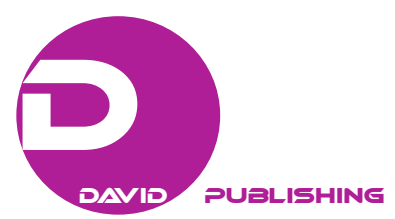

\title{
Asymptotic Inefficiency of Incomplete Asset Markets and Symmetric Event Trees*
}

\author{
Ricardo Luis Chaves Feijó \\ University of São Paulo, Ribeirão Preto, Brazil
}

\begin{abstract}
Demonstrating theoretically the possibility that the financial market, albeit incomplete, has equilibrium and that this equilibrium is efficient and has been an important topic at the frontier of the research on general equilibrium for financial markets. The paper examines the asymptotic properties of incomplete financial markets taking into accounting the asset structure. The paper deals with a case in which a structure of securities relates to the asymptotic inefficiency.
\end{abstract}

Keywords: asymptotic inefficiency, incomplete market, general equilibrium with financial assets, event tree

\section{Introduction}

It seems that the efficient equilibrium in incomplete markets is a contradiction in itself. But it is not so. In fact, in certain contexts, it can be shown that even incomplete financial market may result in equilibrium superior than if the same market has more assets. Superior in the sense is that the utilities of the involved agents are higher than the utilities of the same agents in the case with more assets. This paper proposes to make a critical survey of the literature on general equilibrium with incomplete financial markets that discuss in which condition it is possible to demonstrate the asymptotic properties. In particular, it examines whether each equilibrium that ensues with additions of securities is or is not constrained Pareto optimum (CPO). The constrained optimality analysis runs into the difficulty that it is hard to proceed sensibly without tackling the difficult problem of the determination of the asset structure. With incomplete markets, the asset structures matter. The paper offers and solves an example where a structure of Arrow securities relates to the asymptotic inefficiency.

For this, the paper is organized into three sections, besides the introduction. The first one, a research contest one, discusses the idea of asymptotic inefficiency. The next section, discussion one, argues that the fact that symmetrical two-stage trees generate asymptotic inefficiency it has already been demonstrated in the literature (Mas-Collell, Whinston, \& Green, 1995, p. 712). Nevertheless, the example identifies a particular asset structure that generates this kind of inefficiency in the context. Since in this example, the financial market without securities can be better in terms of welfare, then the condition of efficiency in a restricted set of feasible

\footnotetext{
* Project supported: This research was supported by CNPq-Brazil (National Counsel of Technological and Scientific Development) and FAPESP-São Paulo-Brazil (São Paulo Research Foundation).

Ricardo Luis Chaves Feijó, associate professor, College of Economics, Business Administration and Accounting, University of São Paulo, Ribeirão Preto, Brazil.

Correspondence concerning this article should be addressed to Ricardo Luis Chaves Feijó, Rua Luís Correia de Melo, 250, ap. 1107, Vila Cruzeiro, São Paulo (SP), Brazil, CEP 04626-220.
} 
choices can be achieved here. This weakly notion of optimality is the CPO. Finally, the conclusion section shows the importance of this result to the literature of general equilibrium in incomplete markets, arguing that even if still incomplete market can present optimal welfare properties when considering certain date-event trees for models with two individuals and Arrow securities. A gap is shown in the literature in this direction.

\section{Research Contest}

\section{Definitions}

The main difficulty with the approach is finding a suitable definition of a feasible allocation. Therefore, the present task is to characterize the set of all consumption allocation that can be achieved using existing markets and to investigate whether or not Radner equilibrium (Radner, 1972) is CPO, i.e., Pareto optimal relative to this set. Therefore, it needs a precise description of the constrained feasible set and of the corresponding notion of CPO. There are some proposed solutions. Mas-Collell et al. (1995, p. 711) discussed the definition of this set in the context where there is a single commodity per state. Hart (1975) confined his attention to a subset of the set of all feasible consumption allocations, the subset of competitive equilibria achieved with the same endowment streams. These streams are restricted so that it is possible to move from the original endowment streams to the new endowment streams by redistributing date 1 endowments and reallocating date 1 securities. It is called weakly optimal whether one of these feasible consumptions is not Pareto-dominated by another feasible consumption allocation.

Hart (1975) offered a famous example of the suboptimality of equilibrium in an exchange economy with incomplete markets that last for two periods, where there are two consumers and where there is no uncertainty. Two goods are available in each period. The model assumes that both consumers have the same expectation, and that these expectations turn out to be correct. Inside two Edgeworth boxes, he identified, in each one, the endowments points and analyses four equilibrium points. None of them will be Pareto optimal, however, he showed that one of them is optimal in a weaker sense, that is, Pareto optimal is relative to all allocations that can be achieved by using the existing markets. So in this pioneer essay, Hart (1975) showed by formal model equilibria in incomplete market that even in this weaker sense it is not generally optimal since it may be Pareto-dominated by another, both within the set of feasible allocation. In other words, when markets are incomplete, equilibrium may be Pareto-dominated by another allocation that can be achieved using the same markets. It often occurs in incomplete financial markets however the market structure is complicated.

\section{Asymptotic Inefficiency}

The same author asserts that there is no reason to expect an equilibrium in incomplete market to be even weakly optimal, unless restrictions are imposed in consumers' utility function and endowments. However, the main result obtained by Hart (1975) is what can be deduced from his analysis of the consequences of opening new markets in an incomplete market context. He considered what happens if a new security becomes available at date 1 or date 2. Although the intuition tells that this ought to improve the situation in some sense, Hart (1975) found an example where this is not the case. Each consumer is worse off after the new market has been opened. The author works with two consumers and two specific consumers' utility functions. He arrived at a result that if it applies to an exchange economy situation where there are no transaction costs.

The optimality analysis of equilibria in the incomplete markets economy leads to curious results. In a model in which the set of possible estates of the world is finite, an expanding set of security will, at some finite 
stage, already comprise a complete set. Of course, in this case, it can be sure that a pattern of asymptotic efficiency or asymptotic convergence to an equilibrium situation is occurring that is Pareto optimum when the market is completed. However, in a security market in which uncertainty is described by a countable infinite set of possible states of nature, it is possible that the pattern is the asymptotic inefficiency. If the set of possible states of the world is infinite, the security markets are incomplete remain incomplete at the time. In this case, regardless of the number of securities offered in the market, some wealth patterns cannot be realized as the return on a portfolio of available securities, and equilibrium allocations usually will not be Pareto optimal however it adds more securities. Therefore, there may be a gap between equilibrium and Pareto optimality. It is natural to suppose that this gap would be small if the set of available securities was nearly complete, and would disappear entirely when the number of security is sufficiently large. Notwithstanding, this supposition is wrong in many cases. In fact, all feasible allocation may remain bounded away from Pareto allocations as the number of securities grows. In this case, it has asymptotic inefficiency.

Asymptotic inefficiency far from representing a pathological situation is a typical situation. In the literature, Green and Spear (1987) provided the first example of this phenomenon. Zame (1988) was the first author that identifies a condition in a sequence of securities that is necessary and sufficient for asymptotic efficiency. However, the condition mentioned by him is very strict: every Arrow security should be uniformly approximated by the return on a finite portfolio of the given securities. In fact, most sequence of securities fails these conditions and asymptotic inefficiency is the typical situation. Zame (1990) offered a simple explanation for this recurrent phenomenon in incomplete markets: the requirement that future consumption patterns are non-negative places constraints on the set of portfolios that can be traded. If liabilities of a portfolio were so great that they exceed endowments, satisfying them would violate the requirement that consumption is non-negative and the portfolio cannot is traded. If such portfolios are required in order to implement Pareto optimal trades, feasible security market allocations may be inefficient and remain inefficient even as the set of available securities expands. ${ }^{1}$ Then the asymptotic inefficiency is demonstrated to be a typical case in incomplete financial markets.

Nevertheless, what is this phenomenon? This result clearly reflects the structure of assets that operates in these incomplete markets. However, what is that asset structure? Unfortunately for a non-mathematical economist, most of the mathematical proofs that follow in the literature are based on the use of differential topology. Magill and Shafer (1991, p. 1538) examined this question in terms of individual risk and aggregate risk. If there is no aggregate risk, if both agents face individual risk and if the agents have distinct preferences for the two goods, then a risk-sharing equilibrium cannot be obtained through a system of futures markets. If the asset structure generates risk then in equilibrium, the prices in the two states are linearly independent and the equilibrium exists. If the aggregate risk goes to zero, the prices become more and more collinear, so the agents have to trade more progressively to achieve a given transfer of income. In the limit, no equilibrium exists. In fact, it has absence of equilibria since the spot prices are not sufficiently variable across the states to permit the proper functioning of a system of futures markets. It is reasonable to imagine that the lack of equilibrium is associated with inefficiency. However, the paper's concern is with efficiency analysis between two or more possible equilibria. In general, a similar result can be demonstrated in this context: the asset

\footnotetext{
${ }^{1}$ See Zame (1990). Zame argues that the possibility of default allows that some liabilities may be unsatisfied and this enlarge the set of portfolios that can actually de trade. For him this enlargement is oftentimes in precisely the right directions to lead to efficiency in the markets.
} 
structure in the case of asymptotic inefficiency is such that it must generate no aggregate risk or certainty. Nevertheless, this result is just a particular case, since it has also asymptotic inefficiency for assets structure with aggregate risk.

Debreu (1959) was the first who proposed the interesting special case that occurs when the set of date-event consumption node can be represented as a tree. The event tree reflects the structure of assets traded in the financial market. Zame (1988) identified a condition that is necessary and sufficient for asymptotic efficiency: every Arrow security should be uniformly approximated by the return on a finite portfolio of the given securities. This condition must correspond to a sequence of securities that would be related to specific individual event trees. It is not known a priori how these specific trees would be, but it has been argued here that their combined effect should produce a situation in which there is aggregate risk, since in this case the equilibrium exists and the agents could get better off trading with assets. This is the case of asymptotic efficiency; a rare situation for incomplete markets. It is known that in incomplete markets, the structure of assets that produce asymptotic inefficiency must relate to combinations of individual event tree that would result in the absence of risk in the aggregate. What types of individual trees would be theirs since individual trees can be combined to eliminate the risk? A clear event tree that is candidate is the symmetrical ones. Imagining a game in which at least one of the participants always wins. In this case, there are individual risks, any participant may lose, but there is no collective risk, because someone wins. The purpose of the central theorem of this paper is to show a kind of asset structure applied to a symmetric event tree that produces no risk in the aggregated, if offers a specific and curious example of a kind of asset structure in a symmetric event tree that generates asymptotic inefficiency.

\section{Discussion}

It is argued that it is possible for the number of assets to increase while at the same time everybody becomes worse off at the new incomplete financial market equilibrium. Thus, a common case occurs when it has no aggregate risk. Symmetrical event trees naturally eliminate aggregate risk. Therefore, this is the case considered. Now, it will show a possible asset structure that produces this result through a simple example with just a two-consumer. Following treatment that appears in several essays in the literature on general equilibrium in financial markets, it considers a pure exchange model with simple date-event tree that contains only two periods. In an economy with this simple tree in which $t=0, t=1$, and $t=2$, whether this date-event tree is symmetric, in the sense that it defines bellow, it will find one structure of assets that produces asymptotic inefficiency. Hence, it assumes that there are two goods $1 \in\{1,2\}$ (named goods 1 and 2 ) in each state. For each $i \in\{1,2\}$ and $s \in\{1,2,3,4\}$, let $\omega_{s}^{i} \in R_{++}^{2}$ be the initial endowment of consumer $i$ at state $s$. The existence of asymptotic inefficiency depends crucially on the characteristics of event trees associated with the problem. In the case of symmetric trees, it claims the existence of a specific structure of assets (not the only possible) that eliminates the aggregate risk. For this, it is constructed an economy that ends (at $t=2$ ) with four states. It should explicitly assume that this economy has three dates $t=0,1$, 2. At $t=0$, all four states are indistinguishable. At $t=1$, the first two states $s=1,2$ and the second two states $s=3,4$ become two distinguishable sets. At $t=2$, the uncertainty is completely removed. According to the conventional notation, $T_{0}$ $=\{1,2,3,4\}, T_{1}=\{[1,2],[3,4]\}$, and $T_{2}=\{[1],[2],[3],[4]\}$. Regarding the symmetric tree, in the first two states, the economy behaves according to any normal date-event tree; but in the other four states, the roles of two consumers are reversed. 
This is just a matter of fixing up the necessary notation for all four states (Figures 1 and 2). For two goods $l \in\{1,2\}$, two consumers $i \in\{1,2\}$, and four states in $t=2, s \in\{1,2,3,4\}$. Figures 1 and 2 show the corresponding four date-event trees. The symmetrical two stage data event tree can be constructed in terms of the individual endowments at $t=2$, so it has a formal definition.

Definition 1, taking into account the endowments of consumers at $t=2$, defines symmetrical two stage data-event tree as an event-tree where, for $\omega_{(I s) i}, \omega_{(11) 1}=\omega_{(13) 2}, \omega_{(21) 1}=\omega_{(23) 2}, \omega_{(12) 1}=\omega_{(14) 2}$, and $\omega_{(22) 1}=\omega_{(24) 2}$; $\omega_{(13) 1}=\omega_{(11) 2}, \omega_{(23) 1}=\omega_{(21) 2}, \omega_{(14) 1}=\omega_{(12) 2}$, and $\omega_{(24) 1}=\omega_{(22) 2}$.

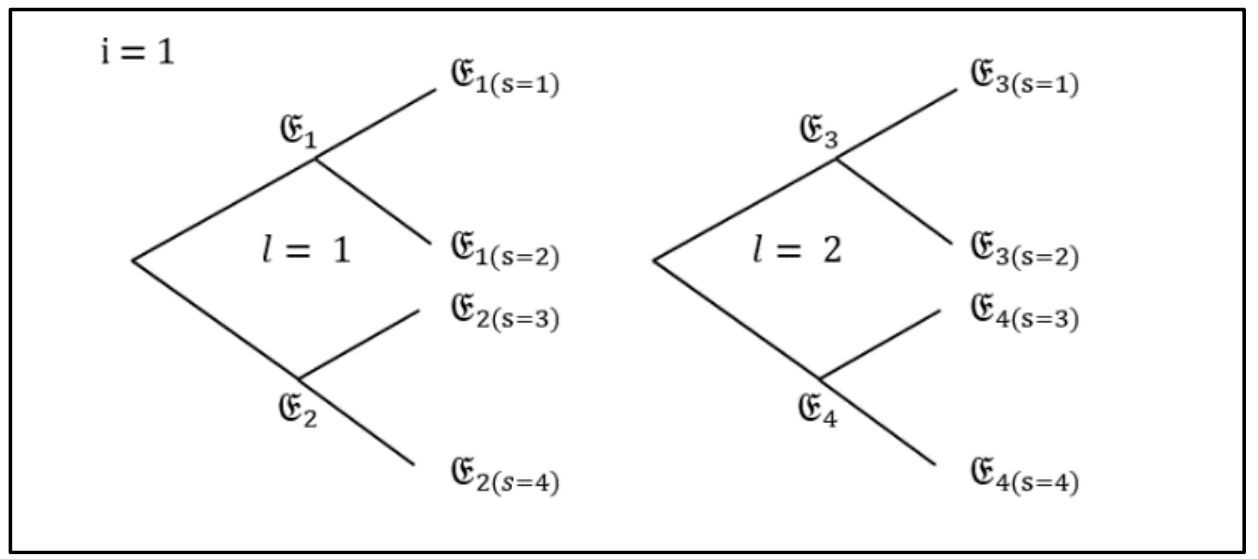

Figure 1. Four data-event trees. Consumer 1. Goods 1 and 2.

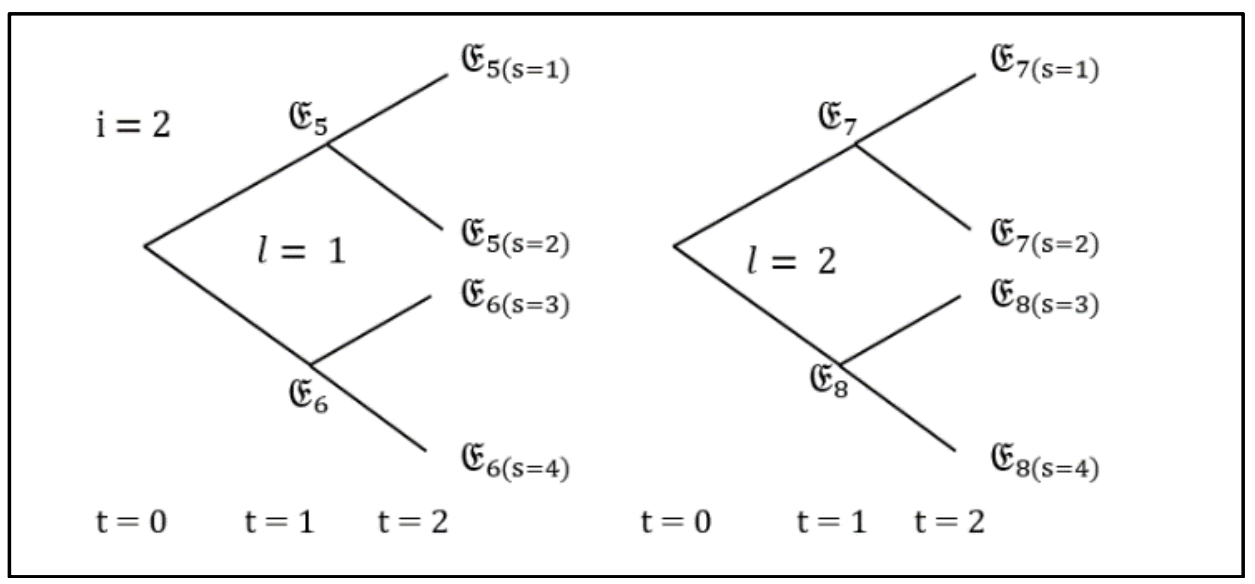

Figure 2. Four data-event trees. Consumer 2. Goods 1 and 2.

Therefore, the endowment on the node $\mathfrak{E}_{6(s=3)}$ equals the endowment on the node $\mathfrak{E}_{1(s=1)}$; the endowment on the node $\mathfrak{E}_{8(s=3)}$ equals the endowment on the node $\mathfrak{E}_{3(s=1)}$; the endowment on the node $\mathfrak{E}_{6(s=4)}$ equals the endowment on the node $\mathfrak{E}_{1(s=2)}$; the endowment on the node $\mathfrak{E}_{8(s=4)}$ equals the endowment on the node $\mathfrak{E}_{3(s=2)}$. Moreover, the endowment on the node $\mathfrak{E}_{2(s=3)}$ equals the endowment on the node $\mathfrak{E}_{5(s=1)}$; the endowment on the node $\mathfrak{E}_{4(s=3)}$ equals the endowment on the node $\mathfrak{E}_{7(s=1)}$; the endowment on the node $\mathfrak{E}_{2(s=4)}$ equals the endowment on the node $\mathfrak{E}_{5(s=2)}$; the endowment on the node $\mathfrak{E}_{4(s=4)}$ equals the endowment on the node $\mathfrak{E}_{7(s=2)}$. These assumptions imply that there is no aggregate uncertainty when considering both individuals. The only uncertainty on the endowment for goods 1 and 2 is simply a swapping between the two consumers across the two states, and on aggregate, there is no uncertainty on this endowment. Then for a simple economy with only two periods and three stages in the event tree, it 
proposes a type asset structure that produces the absence of aggregate uncertainty. First, it needs to find an asset structure to symmetric date-event trees even simpler, with just a single period and two final states of nature. Therefore, it has another formal definition:

Definition 2, taking into account the endowments of consumers at $t=1$, defines symmetrical one stage data-event tree as an event-tree where, for $\omega_{(l s)}, \omega_{(11) 1}=\omega_{(12) 2}$, and $\omega_{(12) 1}=\omega_{(11) 2}$, for contingent commodity 1 , and $\omega_{(21) 1}=\omega_{(22) 1}, \omega_{(21) 2}=\omega_{(22) 2}$ for contingent commodity 2 .

It can explain the meaning of this new definition using four diagrams for the two consumers and two contingent commodities. Let $l$ be the good in question and let $s$ be the state of nature, and $i$ represents the consumer. As showed in Figure 3, for $i=1$, it has in node $\mathfrak{E}_{1}, l=1$, and $s=1$; in node $\mathfrak{E}_{2}, l=1$ and $s=2$; in node $\mathfrak{E}_{3}, l=2$ and $s=1$; and in node $\mathfrak{E}_{4}, l=2$ and $s=2$. Hence, it has four initial endowments $\omega_{(l s) i}: \omega_{(11) 1}$, $\omega_{(12) 1}, \omega_{(21) 1}$, and $\omega_{(22) 1}$.

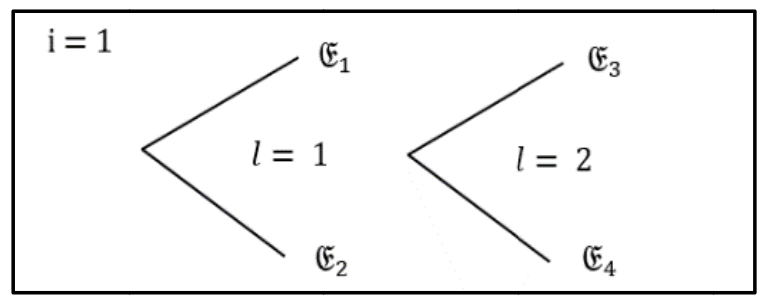

Figure 3. One stage event trees. Consumer 1 . Goods 1 and 2.

In Figure 4, for $i=2$, it has in node $\mathfrak{E}_{5}, l=1$, and $s=1$; in node $\mathfrak{E}_{6}, l=1$ and $s=2$; in node $\mathfrak{E}_{7}, l=2$ and $s=1$; and in node $\mathfrak{E}_{8}, l=2$ and $s=2$. Hence, it has four initial endowments $\omega_{(l s) i}: \omega_{(11) 2}, \omega_{(12) 2}, \omega_{(21) 2}$, and $\omega_{(22) 2}$.

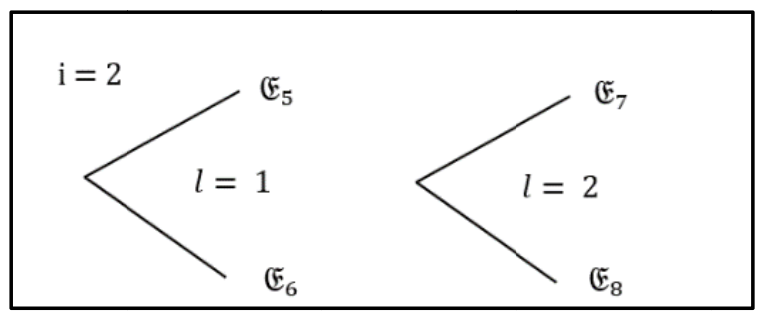

Figure 4. One stage event trees. Consumer 2. Goods 1 and 2.

It assumes that $\mathfrak{E}_{1}=\mathfrak{E}_{6}, \mathfrak{E}_{2}=\mathfrak{E}_{5}$, that is the event-tree that is symmetrical to the first good, in this case, the uncertainty on the endowment for commodity 1 is simply a swapping between the two consumers across the two states; and it assumes that $\mathfrak{E}_{3}=\mathfrak{E}_{4}, \mathfrak{E}_{7}=\mathfrak{E}_{8}$, i.e. there is no uncertainly on the endowment for commodity 2. Naturally, these assumptions imply that there is no aggregate uncertainty. Since there is no aggregate uncertainty concerning the situation of both individuals in the state $t=1$, even for the good 1 given the symmetry of the event tree, it has a fixed endowment for each good $x_{i}$ and a total endowment of these goods expressed in fixed vector $\bar{\omega}=\left(x_{1}, x_{2}\right)$. So let the total endowment vector be $\bar{\omega}$. The only doubt is about the initial distribution of $x_{1 i}$ between two consumers (remembering that there is no uncertainly on the endowment for good 2).

It shall show that in this one-stage tree, a specific type of asset structure result in uncertainty is in the aggregate. For that, it considers Arrow securities contingent first good commodities. It has, therefore, two Arrow securities, one for each consumer. These assets promise to pay the difference between the endowment associated with the state $s$ and the endowment ascribed to the convex combination: $z_{s i}^{* *}=\left[\alpha \omega_{(11) i}+(1-\right.$ 
$\left.\alpha) \omega_{(12) i}\right]-\omega_{(1 s) i}$. This is simply the deviation of the initial endowment of consumer $i$ for good 1 in state $s$ from the convex combination. The Arrow securities ensures that consumers receive at the end the endowment of convex combination associated with good 1 regardless of what is the state of nature $s$.

Noting that $z_{1 i}^{* *}+z_{2 i}^{* *}=\left[\alpha \omega_{(11) i}+(1-\alpha) \omega_{(12) i}\right]-\omega_{(11) i}+\left[\alpha \omega_{(11) i}+(1-\alpha) \omega_{(12) i}\right]-\omega_{(12) i}=$ $(2 \alpha-1)\left(\omega_{(11) i}-\omega_{(12) i}\right)$. Taking into account that for symmetric date-event tree $\omega_{(11) 1}=\omega_{(12) 2}$, $\omega_{(12) 1}=\omega_{(11) 2}$, and so $\omega_{(12) i}=\omega_{(11) j}, i \neq j$, it has $z_{1 i}^{* *}+z_{2 i}^{* *}=(2 \alpha-1)\left(\omega_{(11) i}-\omega_{(11) j}\right)$. If both consumers have the same endowment regarding good 1 on state $1, \omega_{(11) j}=\omega_{(11) i}$ and $z_{1 i}^{* *}+z_{2 i}^{* *}=0$ for $i=$ $\{1,2\}$. Moreover, it has $z_{s 1}^{* *}+z_{s 2}^{* *}=0$ for each $s \in\{1,2\}$, since $z_{s 1}^{* *}+z_{s 2}^{* *}=\left[\alpha \omega_{(11) 1}+(1-\alpha) \omega_{(12) 1}\right]-$ $\omega_{(1 s) 1}+\left[\alpha \omega_{(11) 2}+(1-\alpha) \omega_{(12) 2}\right]-\omega_{(1 s) 2}=\omega_{(12) 1}+\omega_{(12) 2}-\omega_{(1 s) 1}-\omega_{(1 s) 2} \quad$ because $\quad \omega_{(11) 1}=$ $\omega_{(12) 2}$, and $\omega_{(12) 1}=\omega_{(11) 2}$, that is evidently null if $s=2$, and for $s=1$, it is zero because again, it uses $\omega_{(11) 1}=\omega_{(12) 2}$ and $\omega_{(11) 2}=\omega_{(12) 1}$.

It knows from literature that the symmetrical one-stage event tree generates asymptotic inefficiency for this assets structure. ${ }^{2}$ Now it is demonstrated that both Arrow securities are consistent with this phenomenon. Therefore, with them the sum of the utilities at the market equilibrium with Arrow security is smaller than the sum of the utilities at market equilibrium without this security. For symmetrical one-stage event tree, it argues that, in the simple case with only two states of nature, introducing two assets reduces the sum of utilities to each consumer and to both consumers jointly considered, i.e., produces asymptotic inefficiency.

It now considers the case of a date-event tree for two periods and four states of nature like those of Figures 1 and 2 (two goods). Therefore, it now turns to the case with four states of nature. For two periods' date-event tree, it should also specify the asset structure to be introduced as follows: At $t=0$, there is no asset transaction allowed. At $t=1$ and event $\{1,2\}$, the Arrow securities for the first two states $s=1,2$ are traded. At $t=1$ and event $\{1,2\}$, the Arrow securities for the second two states, $s=3,4$ are traded. Define $\left(z_{s i}^{* *}\right): z_{31}^{* *}=z_{12}^{* *}, z_{41}^{* *}=$ $z_{22}^{* *}, z_{32}^{* *}=z_{11}^{* *}, z_{42}^{* *}=z_{21}^{* *}$, suppose that in the symmetrical two stages tree, there is only a single Arrow security that allows the transfer of wealth between two pairs of nodes in $t=1$ : from $\mathfrak{E}_{1}$ and $\mathfrak{E}_{2}$ to $\mathfrak{E}_{3}$ and $\mathfrak{E}_{4}$, for consumer 1 , and from $\mathfrak{E}_{5}$ and $\mathfrak{E}_{6}$ to $\mathfrak{E}_{7}$ and $\mathfrak{E}_{8}$, for consumer 2. Suppose now that four new securities are introduced, one transfers the wealth from $\mathfrak{E}_{1(s=1)}$ to $\mathfrak{E}_{1(\mathrm{~s}=2)}$ and from $\mathfrak{E}_{5(s=1)}$ to $\mathfrak{E}_{5(s=2)}$, other security transfers from $\mathfrak{E}_{3(s=1)}$ to $\mathfrak{E}_{3(s=2)}$ and from $\mathfrak{E}_{7(s=1)}$ to $\mathfrak{E}_{7(s=2)}$, another security transfers the wealth from $\mathfrak{E}_{2(s=3)}$ to $\mathfrak{E}_{2(s=4)}$ and from $\mathfrak{E}_{6(s=3)}$ to $\mathfrak{E}_{6(s=4)}$. Finally, another security transfers wealth from $\mathfrak{E}_{4(s=3)}$ to $\mathfrak{E}_{4(s=4)}$ and from $\mathfrak{E}_{8(s=3)}$ to $\mathfrak{E}_{8(s=4)}$. The asset structure in which there is a single asset allowing a transfer of wealth from the first two states to the other two yields equilibrium that is better for every consumer than the equilibrium obtained if it adds four new assets, one allowing a transfer of wealth between states 1 and 2, other doing the same between states 3 and 4, another transferring wealth between states 5 and 6, and finally other transferring wealth between states 7 and 8. For symmetric two stages date-event tree, if the four Arrow securities are available for trade at $t=1$, then it knows that for each of the two symmetrical branches on $t=1$, each one of these four new securities worsens the utility level of each consumer in the respective branch of the tree in $t=2$. Therefore, the eight nodes to introduce four new assets do not improve the welfare of each of the two consumers. So both consumers get worse off after the introduction of the four Arrow securities at $t=1$. Therefore, also for two stages symmetric date-event tree, it has asymptotic inefficiency.

\footnotetext{
2 See Mas-Collell et al. (1995). Exercise 19.F.3.
} 


\section{Conclusion}

For incomplete markets, the Radner equilibrium not necessarily is a Pareto optimum. In the case of symmetrical trees, the equilibrium with more securities, although a Radner equilibrium, may be inferiored in terms of welfare. It is known that a complete financial market effectively reaches a Radner equilibrium with a Pareto optimum propriety; which means, among other things, the absence of bubbles. Notwithstanding, the result obtained here for the case of symmetric trees shows that some kinds of weakly efficiency could be found in incomplete market. Since the market is asymptotic inefficient, the equilibrium associated with one additional asset (in this case an Arrow security), with it exists, it is Pareto dominated by the previous equilibrium. So the equilibrium achieved by incomplete market is constrained Pareto optimum: the optimality refers to a set of allocations that can be achieved by more than one security. Noting that it does not analyze what happened when it expands the set of securities, i.e., the behavior of equilibrium allocations as the number of assets tends to infinity. Of course, when it completed the financial market, the equilibrium allocations converge to Pareto optimum allocation. Here, it discusses not the proof of financial equilibrium in incomplete markets, but the demonstration of welfare property of each equilibrium as the markets receive a new asset without ever completing, a different context. It remains difficult in demonstrating the equilibrium on financial markets still incomplete. Even when it proved the existence of equilibrium in incomplete financial markets, Paretian efficiency of this equilibrium is not supported, but rather by means of the assumption that the market is really complete. However, in some cases, equilibrium and even efficiency properties can be achieved even for incomplete financial markets. This is the case when you have particular event-trees, such as in the setting here examined for symmetric date-event trees. In short, for two or more agents, the occurrence of symmetrical date-event trees could generate the kind of certainty on aggregate that produces constrained optimum equilibrium, even if the market is incomplete. In short, it may be that the efficient equilibrium exists even for incomplete financial markets, when considering more than one agent, since the event tree has characteristics suitable to this. Of course, postulating the existence of exotic event-trees itself maybe a strong hypothesis, but it is worth investigating different types of trees (trees not just symmetrical as defined in this paper) among market participants for examining the optimal properties of the market. In this paper, it showed the important result that, for certain kinds of trees, the market with most assets produces in an inferior result in welfare, which leaves open the possibility that a Radner equilibrium that is efficiency in some sense, is reached even in incomplete markets. Therefore, it opens a broad avenue of new lines of inquiry to explore the possibility that equilibria that maximize the consumers' welfare in the financial market are achieved while the market is still incomplete (assuming that the market will never be completed). Since in the particular case where the distributional effects of trade are so biased to form symmetrical trees, it is established that with Arrow securities, it has asymptotic inefficiency.

When the date-event trees are symmetrical in the sense defined here, although the number of assets grows, everyone will get worse off in the new equilibrium. It is true that in incomplete markets, in general the Radner equilibrium is Pareto suboptimal, but in some situations, more assets may produce a less desirable outcome for those involved. Therefore, it can be demonstrated (for peculiar date-event trees) that in models of incomplete markets, the equilibrium could have optimum welfare properties. Zame (1988) demonstrated the conditions that must be met for the asymptotic convergence. Since then, it is known that the vast majority of incomplete markets present asymptotic inefficiency, but lacking, in the literature, a more detailed account of the type of 
asset structure and corresponding event trees of the agents that produce certainty on aggregate and therefore asymptotic inefficiency. There still lacks a concrete case worked in detail to demonstrate the association between the absence of aggregate risk and asymptotic inefficiency without the use of an esoterically sophisticated tool as differential topology. A gap is shown in the literature in this direction.

\section{References}

Debreu, A. (1959). Theory of value. New Haven: Yale University Press.

Green, R., \& Spear, S. (1987). Equilibria in large commodity spaces with incomplete financial markets. Journal of Economic Theory, 11, 418-443.

Hart, O. (1975). On the optimality of equilibrium when the market structure is incomplete. Journal of Economic Theory, 11, 419-443.

Magill, M., \& Shafer, W. (1991). Incomplete markets. Chapter 30. In W. Hildenbrand and H. Sonnenschein (Eds.), Handbook of Mathematical Economics, Volume IV.

Mas-Collell, A., Whinston, M. D., \& Green, J. R. (1995). Microeconomic theory. New York-Oxford: Oxford University Press.

Radner, R. (1972). Existence of equilibrium of plans, prices and price expectations in a sequence of markets. Econometrica, 40, 289-303.

Zame, W. R. (1988). Asymptotic behavior of asset markets: Asymptotic inefficiency. Discussion Paper Serie A 220, University of Bonn, Germany.

Zame, W. R. (1990). Efficiency and the role of default when security markets are incomplete. Working Paper 585, University of California. 